University of Nebraska - Lincoln

DigitalCommons@University of Nebraska - Lincoln

$12-1-2000$

\title{
Stabilization of Bound State Decay in an Intense Monochromatic High-Frequency Field
}

\author{
N. L. Manakov \\ Voronezh State University, manakov@phys.vsu.ru \\ M. V. Frolov \\ Voronezh State University, Voronezh, Russia \\ Bogdan Borca \\ Institute for Space Sciences, Bucharest, Romania \\ Anthony F. Starace \\ University of Nebraska-Lincoln, astarace1@unl.edu
}

Follow this and additional works at: https://digitalcommons.unl.edu/physicsstarace

Part of the Physics Commons

Manakov, N. L.; Frolov, M. V.; Borca, Bogdan; and Starace, Anthony F., "Stabilization of Bound State Decay in an Intense Monochromatic High-Frequency Field" (2000). Anthony F. Starace Publications. 123. https://digitalcommons.unl.edu/physicsstarace/123

This Article is brought to you for free and open access by the Research Papers in Physics and Astronomy at DigitalCommons@University of Nebraska - Lincoln. It has been accepted for inclusion in Anthony F. Starace Publications by an authorized administrator of DigitalCommons@University of Nebraska - Lincoln. 


\title{
Stabilization of Bound State Decay in an Intense Monochromatic High-Frequency Field
}

\author{
N. L. Manakov*, M. V. Frolov*, B. Borca**,1, and A. F. Starace** \\ * Voronezh State University, Universitetskaya pl. 1, Voronezh, 394893 Russia \\ ** Department of Physics and Astronomy, The University of Nebraska, Lincoln, NE 68588-0111, USA \\ Received August 8, 2000
}

\begin{abstract}
The formalism of complex quasienergies is used for exact calculation of the field-dependent decay rate for a weakly bound particle (in the model of a three-dimensional zero-range potential) in a strong monochromatic laser field. It is shown that the adiabatic (quasistationary) stabilization regime in this model occurs at frequencies $\omega$ exceeding the binding energy and only in a limited intensity range. A simple estimate is obtained for the critical field of stabilization breakdown. The effect may be observed for the decay of $\mathrm{H}^{-}$ions in the field of a neodymium laser of femtosecond duration. (C) 2000 MAIK "Nauka/Interperiodica”.
\end{abstract}

PACS numbers: $32.80 .-\mathrm{t}$

In spite of the fact that the possibility of the atomic decay rate decreasing with an increase in laser intensity at frequencies higher than the ionization potential was pointed out more than ten years ago $[1,2]$, the physical nature of this interesting nonlinear effect and its dependence on the type of atomic potential and field parameters still remain to be clarified. Essentially, the case in point is a radical modification of the conventional pattern of the photoeffect in a strong field. In [1], the onset of stabilization was associated with a peculiar kind of modification of the atomic potential in an intense high-frequency field, as a result of which the level width $\Gamma$ (imaginary part of the complex quasienergy $\epsilon=\operatorname{Re} \epsilon-i \Gamma / 2$ ) decreases infinitely as the intensity increases, to arrive at the adiabatic (or quasistationary) stabilization (QS). An alternative interference mechanism of QS was proposed for the Rydberg states, where the decrease in $\Gamma$ is caused by destructive interference of the ionization amplitudes of closely spaced levels mixed by a strong field and populated via Raman transitions from the initial state [2]. In recent years, the idea of "dynamic stabilization" (DS) [3] due to the pulsed character of the field has also been actively discussed. Numerical calculations indicate that the "stabilization breakdown" may also occur in ultrastrong pulsed fields [4]. However, the authors of a recent work [5] used the quasistationary quasienergy states (QQESs) as an adiabatic basis in a strong field (see also [6]) to demonstrate that the DS and QS have the same origin. Finally, it was asserted in some works that stabilization is in principle impossible, in particular, QS upon ionization from a short-range potential [7] and DS in pulsed fields [8]. Clearly, these

\footnotetext{
${ }^{1}$ On leave from Institute for Space Sciences, Bucharest, Romania.
}

problems arise because the numerical solution of the initial value problem for the Schrödinger equation in a strong field is a challenge, while analysis of the problem is lacking for exactly solvable analytical models. In this letter, the exactly solvable short-range potential model is taken as an example to analyze the questions of whether the QS regime may occur in the decay of a weakly bound level and, if it does, how large the intensity range for the stabilization is.

We consider quasistationary decay of a bound state in the field of a strong monochromatic wave with electric vector

$$
\begin{gathered}
\mathbf{F}(t)=\frac{F}{\sqrt{1+\eta^{2}}}\{\cos \omega t, \eta \sin \omega t, 0\}, \\
-1 \leq \eta \leq+1
\end{gathered}
$$

and intensity $I=c F^{2} / 8 \pi$ by applying the QQES formalism [9] to the exactly solvable 3D model of a shortrange $(\delta$-) potential having a single bound state with energy $E_{0}[10]$. The exact equation for the complex quasienergy $\epsilon$ contains the degree of linear polarization $l=\left(1-\eta^{2}\right) /\left(1+\eta^{2}\right)$ and the characteristic dimensionless parameters of the problem: $\hbar \omega /\left|E_{0}\right|$ and the ratio of the mean energy of electron oscillations in a field $(e F)^{2} / 4 m \omega^{2}$ to the photon energy $\Delta=(e F)^{2} / 4 m \hbar \omega^{3}$ or to the binding energy $\mathscr{E}_{F}=(e F)^{2} / 4 m \omega^{2}\left|E_{0}\right|=\Delta\left(\hbar \omega /\left|E_{0}\right|\right)$. Below, the following dimensionless units are used: energy and $\hbar \omega$ is in units of $\left|E_{0}\right|$, and field intensity is in units of $F_{0}=\sqrt{4 m\left|E_{0}\right|^{3}} /|e| \hbar$, so that $\mathscr{E}_{F}=F^{2} / \omega^{2}$ and $\Delta=$ 


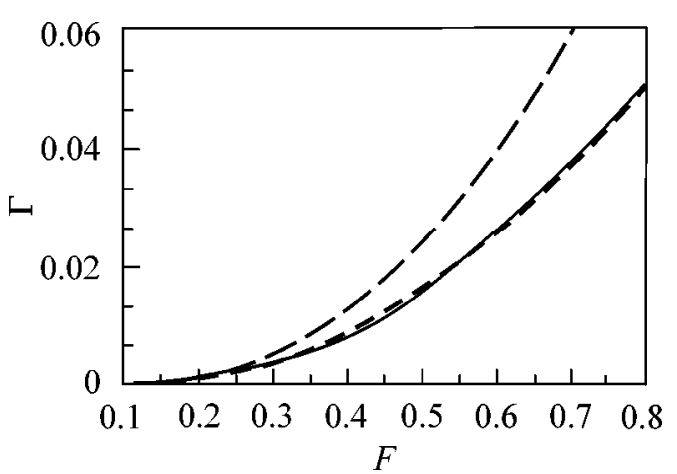

Fig. 1. $\Gamma$ as a function of $F$ for $\omega=0.74$ and circular polarization of laser field. Solid line corresponds to the exact (numerical) calculation according to Eq. (1); dotted line is the result of perturbation theory (3) for $\omega$; dashed line is weak-field approximation (5).

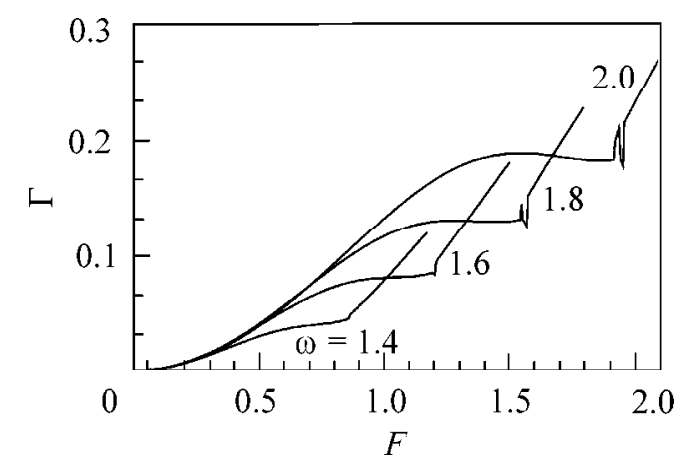

Fig. 2. $\Gamma$ as a function of $F$ for above-threshold frequencies (circular polarization). The $\omega$ values are indicated near the corresponding curves.
$F^{2} / \omega^{2}$ (note that the Keldysh parameter $\gamma=\sqrt{2 m\left|E_{0}\right|} \omega / e F$ in these units is $\gamma=\omega / \sqrt{2} F$ ).

The equation for $\epsilon$ has the simplest form in the case of circularly polarized $\mathbf{F}(t)$ with $\eta= \pm 1$ [11]:

$$
\begin{gathered}
\sqrt{\mathscr{E}_{F}-\epsilon}=1 \\
+\sqrt{\frac{\omega}{4 \pi i}} \int_{0}^{\infty} \frac{\mathrm{d} \tau}{\tau^{3 / 2}} \exp \left\{\frac{-i\left(\mathscr{E}_{F}-\epsilon\right) \tau}{\omega}\right\}\left\{\exp \left[4 i \Delta \frac{\sin ^{2} \tau / 2}{\tau}\right]-1\right\} .
\end{gathered}
$$

For elliptic polarization $(0 \leq|\eta|<1), \epsilon$ is the eigenvalue of a 1D integral equation for a periodic function $\varphi_{\epsilon}(t)$ [which determines the asymptotic behavior of the exact QQES function $\Phi_{\epsilon}(\mathbf{r}, t)$ at $r \longrightarrow 0$ ], with a kernel structurally similar to the integrand in Eq. (1) [10]. Insofar as $\operatorname{Im} \epsilon<0$, the integrals of type (1) formally diverge at the upper limit and thus should be considered in the sense of analytical continuation from the upper $\epsilon$ halfplane. We use the following relation for this analytical continuation:

$$
\int_{0}^{\infty} \frac{\mathrm{d} \tau}{\tau^{1 / 2}} e^{-i \alpha \tau} f(\tau)=\frac{1}{\sqrt{4 \pi i}} \int_{-\infty}^{\infty} \frac{\mathrm{d} k}{\sqrt{\alpha+k}} \int_{-\infty}^{\infty} d \tau e^{i k \tau} f(\tau),
$$

where the double integral converges for any $\alpha=\left(\mathscr{E}_{F}-\right.$ $\epsilon) / \omega$. Note that in some works, where the QQES method was applied to the $\delta$-well model [10], the divergence of the integrals of type (1) was eliminated by substituting $\epsilon \approx E_{0}=-1$, which, clearly, is unjustified for strong fields. In particular, this led the authors of [7] to the erroneous results in their Fig. 5 and to the erroneous conclusion about the absence of QS for short-range potentials.

The numerical results obtained for $\Gamma(F)$ (Figs. 1-3) clearly demonstrate the presence of the QS regime for above-threshold frequencies and abrupt stabilization breakdown, i.e., a sharp increase in $\Gamma(F)$ starting at some critical value $F=F_{c r}$. The width (in $F$ ) of the QS region increases with $\omega$. One can see from Fig. 3 that the $F_{c r}$ value does not depend on the polarization type [see Eq. (7) below], while the $\Gamma(F)$ decrease in the QS region is more pronounced for linear polarization.

Since the problem of stabilization breakdown (and critical field value $F_{c r}$ ) is of crucial importance (in particular, it argues against the existence of the so-called "Death Valley," i.e., a broad and deep minimum in the $F$-dependent lifetime of a quasistationary atomic level [1]), let us make some analytical estimates confirming the numerical results and allowing their physical interpretation. For circular polarization, $\epsilon$ is defined as a (complex) eigenvalue of the stationary Hamiltonian in a coordinate frame rotating with frequency $\omega$ [9],

$$
\mathscr{H}_{r o t}(\mathbf{r})=-\nabla_{\mathbf{r}}^{2}+U(\mathbf{r})+F x \pm \omega \hat{L}_{z},
$$

where $\hat{\mathbf{L}}$ is the orbital angular momentum operator. We will treat the operator $\omega \hat{L}_{z}$ perturbatively and use perturbation theory (PT) in the basis of quasistationary states of a particle in the $\delta$-potential $U(\mathbf{r})=4 \pi \delta(\mathbf{r})(\partial / \partial r)$ and a constant field of strength $\mathbf{F}$. The PT for the quasistationary states is developed, e.g., in [12]. A convenient expression for the Green's function of our problem can be found in [13], where it is expressed through the regular and irregular Airy functions $\operatorname{Ai}(x)$ and $\operatorname{Bi}(x)$ and where the computational technique is also presented for second-order PT. With the $\sim \omega^{2}$ correction, the expression for $\epsilon$ is

$$
\epsilon=E-\frac{\omega^{2}}{360 F^{2 / 3}} \frac{I^{(4)}(\xi)}{I(\xi)}, \quad \xi=-\frac{E}{F^{2 / 3}},
$$

where the complex energy $E$ of a quasistationary state in a field $\mathbf{F}$ is a root of the transcendent equation $\{$ the $E=E(F)$ function is analyzed in [13]\}

$$
1+\pi F^{1 / 3} J\left(-E F^{-2 / 3}\right)=0,
$$




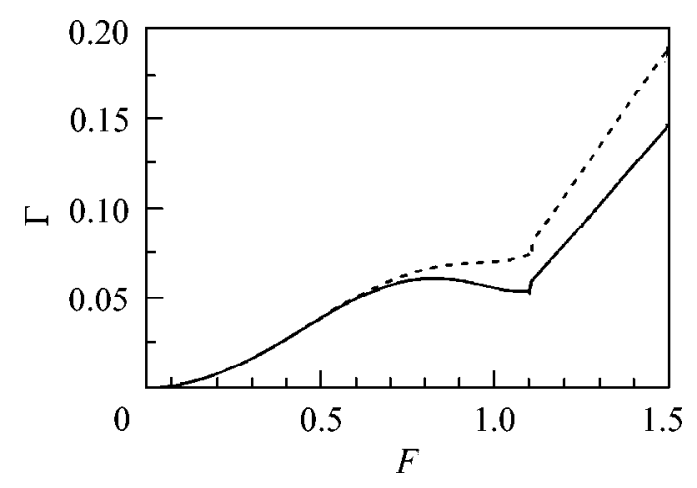

Fig. 3. $\Gamma$ as a function of $F$ for (solid line) linear and (dotted line) circular polarization of a laser field with $\omega=1.55$.

where $J(x)=\mathrm{Ai}^{\prime}(x) \mathrm{Ci}^{\prime}(x)-x \operatorname{Ai}(x) \mathrm{Ci}(x), \mathrm{Ci}(x)=\mathrm{Bi}(x)+$ $i \operatorname{Ai}(x), I(x)=\operatorname{Ai}(x) \operatorname{Ci}(x)$, and $I^{(4)}(x)=\mathrm{d}^{4} I(x) / \mathrm{d} x^{4}$. The $F$ value in Eqs. (3) and (4) is not assumed to be small. Using the properties of the Airy functions and Eqs. (3) and (4), one can easily determine the conditions for applicability of PT to the $\omega \hat{L}_{z}$ operator [i.e., for the smallness of a correction $\sim \omega^{2}$ to the energy $E$ in Eq. (3)].

In the weak-field limit $(F \ll 1)$, one has

$$
\begin{gathered}
\epsilon=-1-\frac{1}{16} F^{2}\left[1+\frac{3}{2} F^{2}+\frac{7}{24}\left(1+13 F^{2}\right) \omega^{2}\right] \\
-\frac{i}{4} F\left[1+\frac{4}{45} \frac{\omega^{2}}{F^{3}}\right] e^{-4 / 3 F} .
\end{gathered}
$$

One can see from Eq. (5) that the perturbative treatment of $\omega \hat{L}_{z}$ is only justified if $\omega$ is small enough $\left(\omega^{2}<F^{3} \ll 1\right)$ that the frequency-dependent correction to the tunneling preexponential factor is small. Note that the corresponding Stark shift $\operatorname{Re} \epsilon+1$ exactly coincides with the two leading terms in the power series expansion of the known expressions for the dynamic polarizability and hyperpolarizability of a weakly bound particle [14]. The inapplicability of expansion (5) at $\omega>1$ is evident, e.g., from the fact that the $F$ dependence of the level width follows a power law. In particular, to the lowest order in $F$, one has $\Gamma_{0}^{(1)}=\left(8 F^{2} / 3 \omega^{4}\right)(\omega-1)^{3 / 2}$ for any polarization of $\mathbf{F}(t)$.

In the $F \gg 1$ limit, Eq. (3) takes the form

$$
\begin{gathered}
\epsilon=0.44133122 e^{-i(\pi / 3)} F^{2 / 3} \\
-F^{1 / 3}\left[0.86328690 e^{-i(\pi / 6)}-0.02548960 e^{i(\pi / 3)} \frac{\omega^{2}}{F^{3}}\right]
\end{gathered}
$$

and demonstrates that the perturbative treatment of $\omega \hat{L}_{z}$ in ultrastrong fields is justified for any frequencies $\omega^{2}<F^{3}$ (curiously, this inequality is the inverse of the condition for applicability of the PT in $F$ to the complex quasienergy [15]: $\Delta \equiv F^{2} / \omega^{3}<1$ ). Although Eq. (6) was derived using the two-term asymptotic expression for energy $E$ obtained in [13] for ultrastrong static fields, result (6) has a fundamental character and confirms (together with the results of direct numerical computations) stabilization breakdown, at least in ultrastrong fields. Note that at $\omega<1$, Eq. (3) agrees well with the exact result even in the region where the PT series in $F$ diverges: starting at $F \sim 0.5$, the exact $\Gamma(F)$ curve for $\omega=0.74$ (Fig. 1) virtually coincides with the curve calculated from Eq. (3). In other words, the action of a strong circularly polarized field at $\omega<1$ is equivalent to the action of a strong static field of strength $\mathbf{F}$. At $\omega>1$, the PT in $\omega \hat{L}_{z}$ applies only to ultrastrong fields, so that in the QS region and at $F \geq F_{c r}$ the results can only be obtained by numerical methods.

The results of numerical and analytical calculations allow one to determine the main regularities of quasistationary level decay in the light field for different ratios between $\left|E_{0}\right|, \omega$, and $F$. At $\Delta \ll 1$, multiphoton decay prevails; i.e., $\Gamma \sim F^{2 N}$ with $N=\left[\left|E_{0}\right| / \omega\right]$ for any $F$ and $\omega$, including $\omega \ll 1$. As $F$ increases, the situation qualitatively depends on the frequency: for small $\omega$, the value $\Delta \sim 1$ is attained in a relatively weak field $F$ and the perturbative decay regime is smoothly replaced by tunneling (according to Keldysh). This mechanism is operative in fields much weaker than the intraatomic fields [see Eq. (5) and the dashed line in Fig. 1]. It was considered in detail in [16] for arbitrary values of the Keldysh parameter $\gamma$. At $\omega>1$, the multiphoton ionization mechanism prevails even in fields for which the lowest order PT $\left(\Gamma_{0}^{(1)}\right)$ does not apply and the higher order corrections to $\Gamma_{0}^{(1)}$, caused by reemission and direct above-threshold photon absorption, should be taken into account. However, for these $F$ values, the linear dependence of $\Gamma$ on the intensity is replaced by a smoother dependence (the $\sim F^{2}$ correction to the width $\Gamma_{0}^{(1)}$ is negative [14]) and tunneling is replaced by the QS regime starting at fields $F<\omega$ for which the highorder PT corrections become significant. Evidently, the standard PT, with $F$ as a small parameter, does not apply in the range of "developed" stabilization.

Turning to the stabilization breakdown point $F_{c r}$, note that it is preceded by a (rather narrow) range of fields $F \leq F_{c r}$ where the width $\Gamma$ suffers irregular jumps with relatively small amplitudes (Fig. 2; the exact numerical calculation of $\Gamma$ in this region presents considerable difficulty). To perform a more detailed analysis of the level width in the breakdown region, we calculated nonperturbatively the partial widths $\Gamma^{(n)}(F)$ corresponding to the absorption of a fixed number $n$ of photons. Having no room for a detailed discussion, we merely point out that the "one-photon" width $\Gamma^{(1)}$ (corresponding to the photoelectron energy $E_{p}=\operatorname{Re} \epsilon-\mathscr{E}_{F}+\omega$ and almost completely determining the total width $\Gamma$ up 
to the middle of the QS interval) has a deep minimum in the breakdown region, so that the contribution to $\Gamma$ in the breakdown region comes from a large number of (interfering) above-threshold $n$-photon decay channels. We assume that the irregular $\Gamma(F)$ dependence in this narrow range of $F$ values is a quantum manifestation of the well-known chaotic behavior typical of classical systems with strong nonlinearity at a certain ratio of relevant parameters (for the application of the ideas of dynamic chaos to the stabilization problem, see, e.g., [17]). Assuming that the minimum in the one-photon width $\Gamma^{(1)}$ is caused by closing the direct photoionization channel, one can estimate $F_{c r}$ from the equality $\operatorname{Re} \epsilon-\mathscr{E}_{F}+\omega=0$. Neglecting the Stark shift Re $\epsilon+1$, which is small at $\omega \sim 1$, one has

$$
F_{c r} \approx \omega \sqrt{\omega-1} .
$$

This estimate agrees nicely with the $F_{c r}$ value obtained by numerical calculations for frequencies up to $\omega \leq 3$; a slight decrease [compared to Eq. (7)] in $F_{c r}$ with increasing $\omega$ is due to the neglect of the Stark shift. Therefore, both the specific behavior of $\Gamma(F)$ in the QS region and the occurrence of this region and its breakdown are caused by a profound modification of the threshold phenomena for the photoeffect in a strong monochromatic field.

Note in conclusion that the analysis carried out in this work gives evidence for the presence, in a limited intensity range, of the QS regime in the quasistationary decay of a weakly bound state in a strong field with frequency higher than the binding energy and also provides a simple estimate for the QS breakdown point $F_{c r}$. The $\delta$-potential model adequately describes photoprocesses in negative ions, in particular, $\mathrm{H}^{-}$(with $\left|E_{0}\right| \approx$ $0.752 \mathrm{eV}$ ). For $\mathrm{H}^{-}$, the frequency of the neodymium laser equals $\omega_{\mathrm{Nd}} \approx 1.55$ and stabilization is possible (Fig. 3) in fields $F \sim 1\left(I \approx 3 \times 10^{12} \mathrm{~W} / \mathrm{cm}^{2}\right)$. Since the lifetime of $\mathrm{H}^{-}$in this field is rather short, $\tau \sim 1 / \Gamma \sim 10 \mathrm{fs}$, stabilization can be observed only in experiments with femtosecond laser pulses.

We are grateful to M.V. Fedorov for helpful discussions. This work was supported in part by the INTASRFFI (grant no. 97-673), the Russian Foundation for Basic Research (project no. 00-02-17843), and the NSF (grant no. PHY-0070980).

\section{REFERENCES}

1. M. Pont and M. Gavrila, Phys. Rev. Lett. 65, 2362 (1990); M. Gavrila, in Photon and Electron Collisions with Atoms and Molecules, Ed. by P. G. Burke and C. J. Joachain (Plenum, New York, 1997), p. 47.

2. M. V. Fedorov and A. M. Movsesian, J. Phys. B 21, L155 (1988); M. V. Fedorov, Laser Phys. 9, 209 (1999).

3. Q. Su, Laser Phys. 2, 241 (1993); Q. Su, B. P. Irving, C. W. Johnson, and J. H. Eberly, J. Phys. B 29, 5755 (1996).

4. Q. Su, B. P. Irving, and J. H. Eberly, Laser Phys. 7, 1 (1997); A. Patel, N. J. Kylstra, and P. L. Knight, J. Phys. B 32, 5759 (1999); A. M. Popov, O. V. Tikhonova, and E. A. Volkova, Laser Phys. 10, 779 (2000).

5. M. Dorr and R. M. Potvliege, J. Phys. B 33, L233 (2000).

6. H. C. Day, B. Piraux, and R. M. Potvliege, Phys. Rev. A 61, 031402 (2000).

7. V. P. Kraŭnov and M. A. Preobrazhenskiŭ, Zh. Éksp. Teor. Fiz. 103, 1142 (1993) [JETP 76, 559 (1993)].

8. S. Geltman, J. Phys. B 32, 853 (1999); T. Mercouris and C. Nicolaides, J. Phys. B 32, 2371 (1999); C. Figueira, A. Fring, and R. Schrader, Laser Phys. 9, 379 (1999).

9. N. L. Manakov, V. D. Ovsiannikov, and L. P. Rapoport, Phys. Rep. 141, 319 (1986).

10. N. L. Manakov and A. G. Fănnshtern, Dokl. Akad. Nauk SSSR 244, 567 (1979) [Sov. Phys. Dokl. 24, 41 (1979)]; Zh. Éksp. Teor. Fiz. 79, 751 (1980) [Sov. Phys. JETP 52, 382 (1980)].

11. N. L. Manakov and L. P. Rapoport, Zh. Éksp. Teor. Fiz. 69, 842 (1975) [Sov. Phys. JETP 42, 430 (1975)]; I. J. Berson, J. Phys. B 8, 3078 (1975).

12. Ya. B. Zel'dovich, Zh. Éksp. Teor. Fiz. 39, 776 (1961) [Sov. Phys. JETP 12, 542 (1961)].

13. N. L. Manakov, M. V. Frolov, A. F. Starace, and I. I. Fabrikant, J. Phys. B 33, R141 (2000).

14. N. L. Manakov, M. A. Preobrazhenskiŭ, L. P. Rapoport, and A. G. Fănnshteŭn, Zh. Éksp. Teor. Fiz. 75, 1243 (1978) [Sov. Phys. JETP 48, 626 (1978)].

15. N. L. Manakov and A. G. Fănnshteŭn, Teor. Mat. Fiz. 48, 375 (1981); R. M. Potvliege and R. Shakeshaft, Phys. Rev. A 41, 1609 (1990).

16. L. V. Keldysh, Zh. Éksp. Teor. Fiz. 47, 1945 (1964) [Sov. Phys. JETP 20, 1307 (1964)]; A. I. Nikishov and V. I. Ritus, Zh. Éksp. Teor. Fiz. 50, 255 (1966) [Sov. Phys. JETP 23, 168 (1966)]; A. M. Perelomov, V. S. Popov, and M. V. Terent'ev, Zh. Éksp. Teor. Fiz. 50, 1393 (1966) [Sov. Phys. JETP 23, 924 (1966)]; Zh. Éksp. Teor. Fiz. 51, 309 (1966) [Sov. Phys. JETP 24, 207 (1967)].

17. W. Chism, D. I. Choi, and L. E. Reichl, Phys. Rev. A 61, 054702 (2000).

Note added in proof: We have recently become aware of a related paper by P.S. Krstic et al., Phys. Rev. A 44, 3089 (1991).

Translated by V. Sakun 